\title{
Analysis of Morphologic and Hemodynamic Parameters for Unruptured Posterior Communicating Artery Aneurysms with Oculomotor Nerve Palsy
}

\author{
Y. Yu, J. Xu, Y. Fang, X. Wu, P. Yang, C. Jiang, Y. Qian, J. Liu, and Q. Huang
}

\begin{abstract}
BACKGROUND AND PURPOSE: Posterior communicating artery aneurysms with oculomotor nerve palsy may imply sudden enlargement of the aneurysm sac and have a high risk of rupture. Our aim was to identify the morphologic and hemodynamic parameters in this special period of aneurysm progression and to assess related rupture risk indices.
\end{abstract}

MATERIALS AND METHODS: We analyzed the morphologic and hemodynamic parameters of 9 unruptured posterior communicating artery aneurysms with oculomotor nerve palsy and 9 ruptured ones. The morphologic parameters were measured and calculated from patient-specific 3D rotational angiographic images, and pulsatile computational fluid dynamic simulation was then performed for hemodynamic parameters.

RESULTS: There was no significant statistical difference between the 2 groups in size, aspect ratio, size ratio, aneurysm angle, or vessel angle; analysis only demonstrated a significantly lower wall shear stress of the aneurysm wall in the symptomatic unruptured group in hemodynamics $(P=.024)$, whereas there were no differences in wall shear stress of the parent artery, low wall shear stress area, and oscillatory shear index.

CONCLUSIONS: From morphologic and hemodynamic perspectives, we demonstrated that posterior communicating artery aneurysms with oculomotor nerve palsy had characteristics similar to those of ruptured ones, except for lower wall shear stress on the aneurysm wall, which might indicate an important role in aneurysm rupture.

ABBREVIATIONS: $\quad$ A = intracranial aneurysm; PcomA = posterior communicating artery; $C F D=$ computational fluid dynamics; $A R=$ aspect ratio; $S R=$ size ratio; WSS = wall shear stress; LSA = low wall shear stress area; OSI = oscillatory shear index

$\mathbf{R}$ upture risk evaluation for an unruptured intracranial aneurysm (IA) is the critical factor for clinical decision-making. Many studies using image-based computational fluid dynamics (CFD) to evaluate rupture risk for unruptured IAs by hemodynamic and morphologic characteristics have been reported, however, most compared findings with ruptured IAs. ${ }^{1,2}$ The CFD

Received December 2, 2012; accepted after revision February 15, 2013

From the Department of Neurosurgery (Y.Y., J.X., Y.F., X.W., P.Y., C.J., J.L., Q.H.), Changhai Hospital, Second Military Medical University, Shanghai, China; and Australian School of Advanced Medicine (Y.Q.), Macquarie University, Sydney, Australia.

Y. Yu and J. Xu are co-first authors.

This study was supported by National Natural Science Foundation of China (Grant No.81171092, J. Liu; Grant No.81171093, Q. Huang; Grant No. 81000494, P. Yang); Rising-star Plan and Key Project of Shanghai Science and Technology Committee, China (Grant No. 11QA1408400, Q. Huang; Grant No. 11JC1415800, J. Liu); the Dawn Project of Shanghai, China (Grant No. 11CG043, P. Yang); Australian Research Council (Grant Nos. DP110102985 and LP0990263, Y. Qian).

Please address correspondence to Qinghai Huang, MD, Department of Neurosurgery, Changhai Hospital, 168 Changhai Rd, Shanghai, 200433, China; e-mail: ocinhqh@163.com

- Indicates open access to non-subscribers at www.ajnr.org

http://dx.doi.org/10.3174/ajnr.A3575 study of ready-to-rupture IAs, which is very difficult in clinical data acquisition, should be more reasonable and revealing but is less practical. Oculomotor nerve palsy occurring in unruptured posterior communicating artery (PcomA) aneurysms is considered a result of the pulsatile compression of sudden enlargement of the aneurysm sac. ${ }^{3,4}$ Neurosurgeons and neurointerventionalists agree that a PcomA aneurysm with oculomotor nerve palsy is at high rupture risk, and it is an indication for urgent treatment. The hemodynamic and morphologic characteristics of this special type of IA with sudden enlargement should be similar to ready-to-rupture aneurysms, and the differences from ruptured PcomA aneurysms would more accurately indicate the hemodynamic and morphologic characteristics facilitating IA rupture. Thus, our aim was to identify the morphologic and hemodynamic parameters in this special period of aneurysm progression and to assess related rupture risk indices.

\section{MATERIALS AND METHODS Patient Population}

The medical records and 3D angiography data of patients with IAs that were diagnosed and treated in our department for the period 


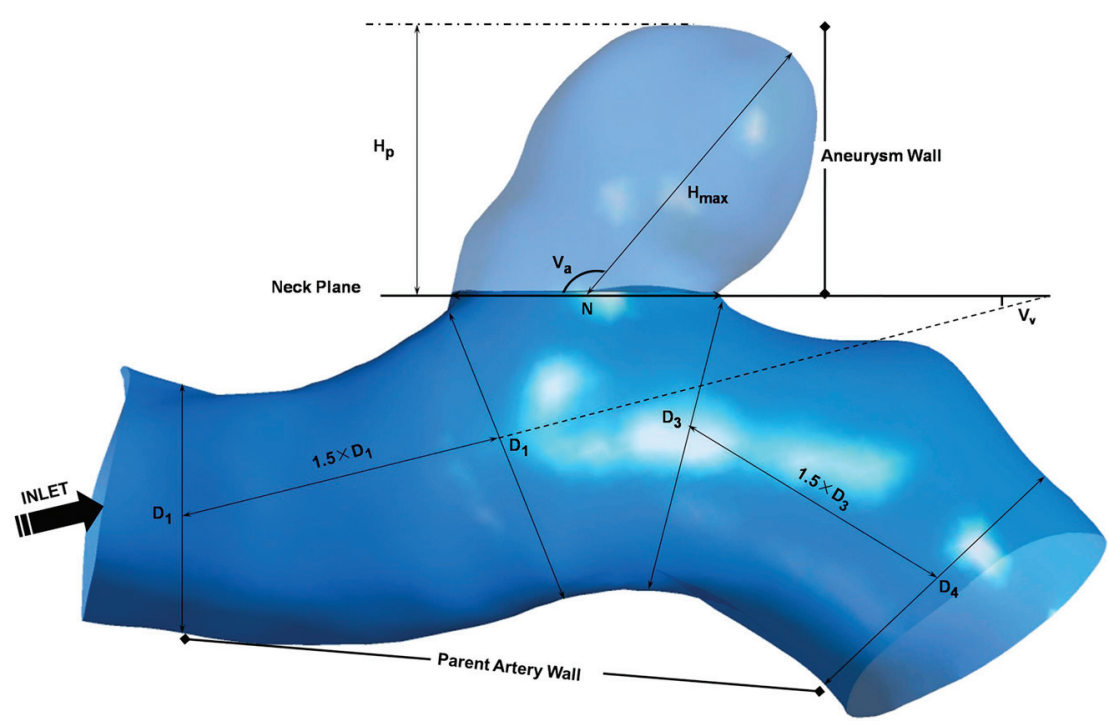

FIG 1. Measurement of morphologic parameters and division of the vessel wall. Size $=H_{p} ; A R=$ $H_{p} / N ; S R=H_{\text {max }} / D_{m}$, where $D_{m}=\left(D_{1}+D_{2}\right) / 2$

2006-2012 were reviewed. Only patients with unruptured PcomA aneurysms who had sudden homolateral oculomotor nerve palsy and whose $3 \mathrm{D}$ data were of sufficient quality for further CFD study were included. Nine aneurysms from 9 patients were used in this study. The mean age in this case series was 65.7 years, with a range of $37-82$ years. The time interval between the onset of first symptom and admission was 14.3 days on average. The control group consisted of 9 patients with angiographically confirmed ruptured PcomA aneurysms that were chosen randomly from the case library. The institutional review board in our hospital approved this study.

\section{DSA Image Acquisition and Hemodynamic Modeling}

Patient-specific 3D rotational angiographic images were obtained by standard transfemoral catheterization of the cerebral vessels with the use of Allura Xper FD20 (Philips, Best, the Netherlands). Stereolithography format files were imported into the software ICEM CFD (ANSYS, Canonsburg, Pennsylvania) to generate volumetric finite-element grids after $3 \mathrm{D}$ reconstruction on a $3 \mathrm{D}$ workstation (Philips). The number of total elements of each vessel luminal model was approximately 550,000-800,000. We defined the vessel wall as divided into 3 parts: aneurysm wall, parent artery wall, and other vessel wall (Fig 1).

CFX 11.0 (ANSYS) was used for CFD simulation. We simulated 3 continuous 0.8 -second pulsatile cardiac cycles. ${ }^{5}$ There are 800 time-steps in each cycle. Blood was considered an incompressible Newtonian fluid with attenuation $\rho=1.0 \mathrm{~g} / \mathrm{cm}^{3}$ and viscosity $\mu=0.035$ poise and modeled on the basis of the unsteady Navier-Stokes equations. A traction-free boundary condition was imposed to the outlets. The vessel wall was assumed to be rigid with no-slip boundary conditions.

After simulation, the time-averaged wall shear stress (WSS) of the aneurysm wall and parent artery wall over the second cardiac cycle were calculated. Low wall shear stress area (LSA) was defined as the area of the aneurysm wall where the WSS is $<10 \%$ of the mean parent arterial $\mathrm{WSS}^{5-7}$ and then normalized by dome area ${ }^{7}$. Oscillatory shear index (OSI) was calculated by the formula de- scribed by $\mathrm{He}$ et $\mathrm{al}^{8}$ and then was also averaged over the dome area.

\section{Morphologic Parameter Calculations}

Morphologic metrics were calculated from 3D angiographic data obtained and reconstructed as described previously by 2 individuals (Y.Y. and J.X.), and their average values were obtained. All the morphological parameters such as aneurysm size, aspect ratio (AR), size ratio $(\mathrm{SR})$, aneurysm angle $\left(\mathrm{V}_{\mathrm{a}}\right)$, and vessel angle $\left(\mathrm{V}_{\mathrm{v}}\right)$ were defined and calculated as described in the study performed by Dhar et $\mathrm{al}^{9}$ (Fig 1). Specifically, because most vessels in patients bend in all 3 dimensions, the values of these morphologic parameters depend on the direction from which the geometry is viewed. The correct viewing plane must be determined as follows before measuring: Aneurysm neck plane is kept parallel with the viewing plane, and the geometry is rotated about the axis that passes through the neck centroid and is perpendicular to the neck plane until the value of apparent vessel angle is minimum. ${ }^{9}$ We considered all the aneurysms to be side wall aneurysms and ignored the effects of posterior communicating artery that are $<0.5 \mathrm{~mm}$ in diameter on morphologic metrics.

\section{Statistical Analysis}

First, normality and homogeneity of variance tests were performed by application of Shapiro-Wilk and Levene statistics to all the data, respectively. Considering the small sample size, for data that conformed to both the normality and homogeneity of variance, a 2-tailed Student $t$ test was performed to check the statistical significance of the mean difference between the unruptured and ruptured groups, and a nonparametric Mann-Whitney $U$ test was used for the assessment of other parameters. Both methods are adequate for small sample size. We hope that proper statistical methods reduce the probability of type 2 error caused by small sample size and the conclusions are reasonable. The $P$ value was calculated for each test, and significance was assumed as $P<.05$.

\section{RESULTS}

All results are detailed in the Table. Figs 2 and 3 depict the contour of time-averaged WSS and OSI. Because most parameters did not conform to both the normality and homogeneity of variance tests, we then performed a statistical analysis of all the parameters by use of the Mann-Whitney $U$ test. All results are recorded as median $(25 \%$ percentile, $75 \%$ percentile) or median \pm interquartile range. The symptomatic unruptured PcomA aneurysms were very similar to ruptured ones in morphology and hemodynamics. There was no significant statistical difference between the 2 groups in any morphologic parameters, such as $V_{a}, V_{v}$, size, $A R$, and $S R$. AR in the symptomatic unruptured group was slightly higher than that in the ruptured group $(1.35 \pm 1.12$ versus $0.97 \pm 0.49, P=.050)$. We 
demonstrated a lower WSS in the symptomatic unruptured group $(5.27 \pm 3.20$ versus $8.11 \pm 4.93, P=.024)$. For other hemodynamic parameters, including WSS of the parent artery wall, LSA, and OSI, there were no significant differences between the 2 groups.

\section{DISCUSSION}

With the development of CFD techniques, more neurointerventionalists are realizing their clinical utility and are trying to use

Statistical analysis of morphologic and hemodynamic parameters

\begin{tabular}{llcl}
\hline & $\begin{array}{c}\text { Symptomatic } \\
\text { Unruptured } \\
(\boldsymbol{n}=9)\end{array}$ & $\begin{array}{c}\text { Ruptured } \\
(\boldsymbol{n}=9)\end{array}$ & $\begin{array}{c}\boldsymbol{P} \\
\text { Value }\end{array}$ \\
\hline $\mathrm{V}_{\mathrm{a}}$ & $111.61(85.30,125.60)$ & $101.20(65.42,125.15)$ & .796 \\
$\mathrm{~V}_{\mathrm{v}}$ & $11.50(9.77,17.05)$ & $11.50(6.65,30.65)$ & .730 \\
$\mathrm{Size}$ & $5.73(4.11,8.97)$ & $5.35(4.63,6.03)$ & .546 \\
$\mathrm{AR}$ & $1.35(0.99,2.14)$ & $0.97(0.84,1.33)$ & .050 \\
$\mathrm{SR}$ & $1.94(1.51,3.30)$ & $1.74(1.57,2.53)$ & .489 \\
WSS of aneurysm & $5.27(3.11,6.31)$ & $8.11(5.57,10.50)$ & .024 \\
$\quad$ wall & & & \\
WSS of parent & $11.58(9.13,12.57)$ & $13.06(11.48,17.02)$ & .077 \\
$\quad$ artery wall & & & \\
LSA & $0.100(0.001,0.278)$ & $0.006(0.002,0.667)$ & .258 \\
OSI & $0.035(0.008,0.054)$ & $0.015(0.009,0.042)$ & .666 \\
\hline
\end{tabular}

Note:-All results are recorded as median (25\% percentile, $75 \%$ percentile); unit of $V_{a}$ and $V_{v}$ is degree; unit of size is cm; unit of WSS is $\mathrm{Pa}$.

$\mathrm{V}_{\mathrm{a}}$ indicates aneurysm angle; $\mathrm{V}_{\mathrm{v}}$, vessel angle; $A S$, aneurysm size. these powerful tools to guide neurointerventional practice in treating IAs. The morphologic and hemodynamic differences between ruptured and unruptured aneurysms were compared among different individuals to evaluate the rupture risk of IAs. ${ }^{9-13}$ However, most of time, we only obtain the postrupture details of an aneurysm and its parent artery. Once an aneurysm ruptures, sudden expansion of a pseudosac or ruptured points may cause great change in intra-aneurysmal hemodynamic environment and mitigate the blood flow to stabilize the ruptured aneurysm. Accordingly, the best way to evaluate the rupture risk of IA is to study the aneurysm in the ready-to-rupture state. A few cases of gross blood-flow characteristics of IA just before rupture have been reported, ${ }^{14,15}$ but without comparison with ruptured aneurysms. The exact morphology and hemodynamics just before aneurysm rupture are not clear yet, but both studies of aneurysms before their rupture demonstrated low WSS on the aneurysm sacs. Some studies revealed lower WSS on ruptured aneurysms than on asymptomatic unruptured ones. $5,7,16$ A CFD study of hemodynamic differences between unruptured and ruptured IAs during observation has been reported recently. Thirteen aneurysms that ruptured during the course of follow-up observation were included in this study, and averaged WSS in the unruptured group was lower than in the rup-

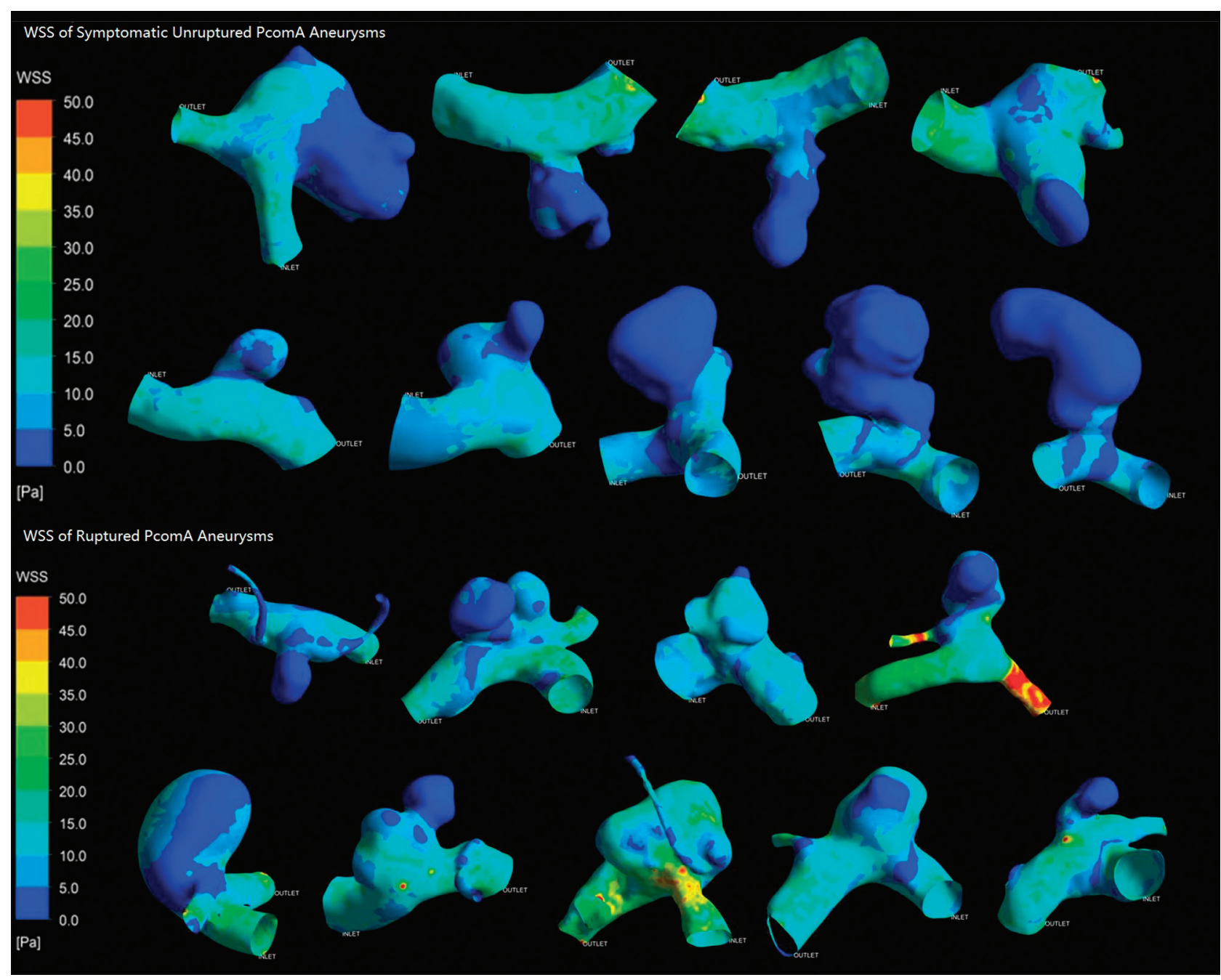

FIG 2. Wall shear stress of symptomatic unruptured and ruptured posterior communicating artery aneurysms. 

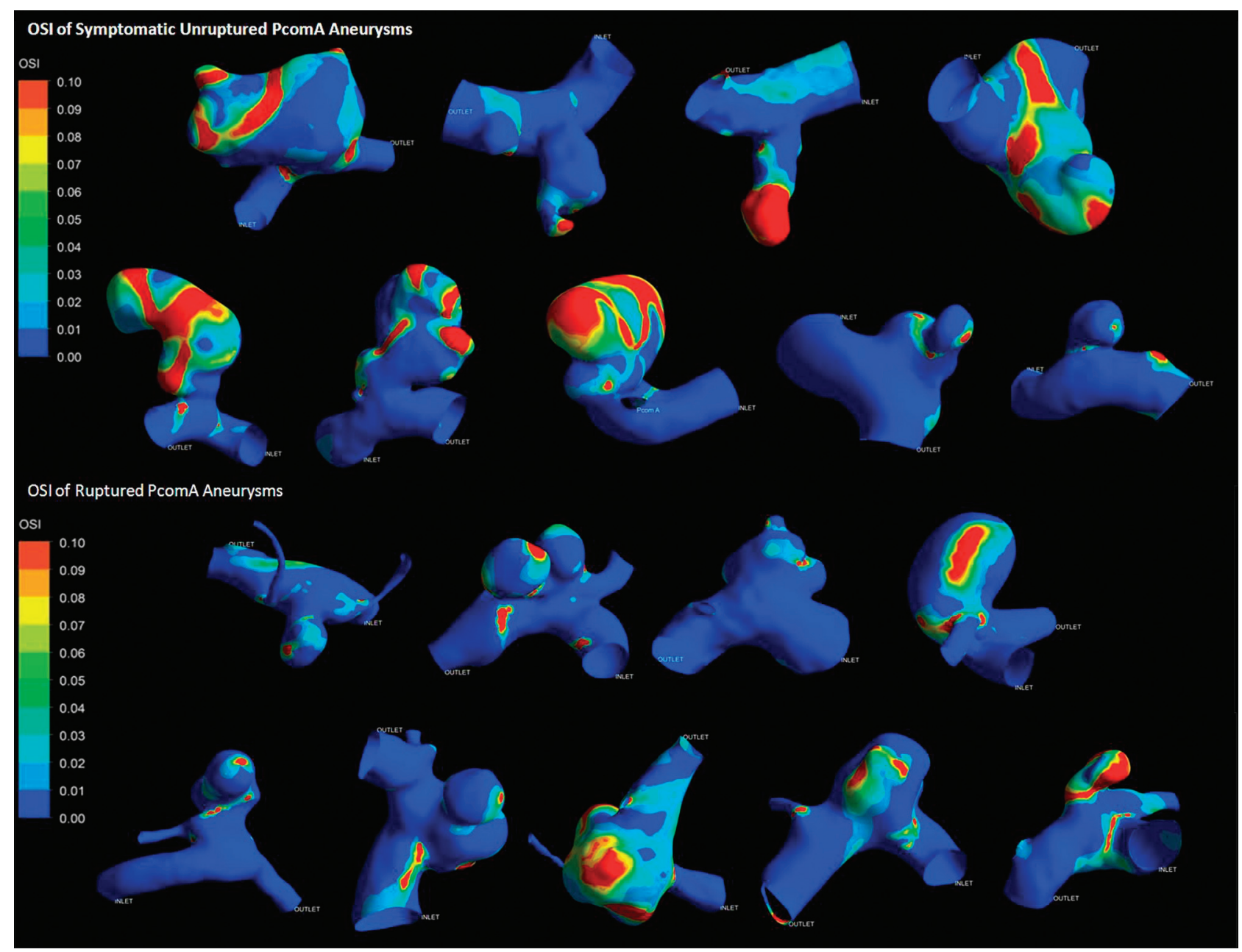

FIG 3. Oscillatory shear index of symptomatic unruptured and ruptured posterior communicating artery aneurysms.

tured group in the MCA. In our study, we also noticed a lower WSS in the unruptured PcomA aneurysms with oculomotor nerve palsy than in the ruptured ones $(5.27 \pm 3.20$ versus $8.11 \pm 4.93, P=.024)$, which is similar to the qualitative trend in the Takao et al study. ${ }^{17}$ Also, the AR was larger in the symptomatic unruptured group, and the $P$ value of AR was very close to statistical significance $(1.35 \pm 1.12$ versus $0.97 \pm 0.49$, $P=.050)$. This means that compared with ruptured PcomA aneurysms, symptomatic unruptured ones have a more irregular shape. Destructive remodeling may take place, which facilitates IA rupture caused by pathobiologic responses induced by low WSS, such as matrix metalloproteinase production by macrophages, ${ }^{18,19}$ increased inflammatory cell infiltration, ${ }^{20}$ and increased reactive oxygen species. ${ }^{21}$ A recent study performed on an animal model also proved that high AR appears more deleterious to the aneurysm wall with pathologically low WSS. $^{22}$ Above all, in this case series, it inferred that low WSS might play an important role in aneurysm rupture.

In another study we performed, we analyzed morphologic and hemodynamic parameters of mirror PcomA aneurysms. ${ }^{23}$ We found that lower WSS, higher LSA, and higher OSI contribute to aneurysm rupture. However, our results in this study only demonstrated that WSS of the aneurysm wall was significantly different between the 2 groups, instead of LSA or OSI hemodynamically. This discrepancy may be caused by small sample size, and it may reveal the particularity of these special symptomatic aneurysms in some ways. We should note that all unruptured aneurysms in the previous study were asymptomatic ones. This indicates that PcomA aneurysms with oculomotor nerve palsy, such as symptomatic IAs, might be different from asymptomatic unruptured or ruptured ones, which deserve separate study. Oculomotor nerve palsy is an indication for urgent treatment. This consensus is mainly based on vast clinical observations and physician clinical experience. Statistical analysis of morphologic and hemodynamic parameters revealed that unruptured PcomA aneurysms with oculomotor nerve palsy had almost the same morphologic and hemodynamic characteristics as that of ruptured ones. From this point of view, urgent treatment should be reasonable to prevent severe consequences after aneurysm rupture.

The small sample size is due to the fact that a PcomA aneurysm that has sudden unilateral oculomotor nerve palsy before it ruptures is relatively rare. That was the main limitation of this study. Larger case series are needed for further study and to generalize the result. As a retrospective study, we could not use the patientspecific boundary conditions to perform the CFD simulation. The same and specified initialization settings and inlet boundary conditions made hemodynamic results depend on the geometry of the luminal models. Also, traction-free outlet boundary conditions were another limitation to this study, but proper assump- 
tions of outlet boundary conditions must be further investigated. ${ }^{7,24}$ In some cases, it was arbitrary to ignore the influence of the poorly developed posterior communicating artery, the diameter of which was $<0.5 \mathrm{~mm}$.

\section{CONCLUSIONS}

From morphologic and hemodynamic perspectives, we demonstrated that PcomA aneurysms with oculomotor nerve palsy had characteristics similar to those of ruptured ones, except for lower WSS on the aneurysm wall, which might indicate an important role in aneurysm rupture.

\section{ACKNOWLEDGMENTS}

We gratefully acknowledge Shanghai Supercomputer Center for their helpful provision of the calculation software. We are also grateful for the generous support of Jianping Xiang from Toshiba Stroke Research Center, State University of New York at Buffalo, Buffalo New York; Shengzhang Wang, from the Department of Mechanics and Engineering Science, Fudan University, Shanghai, China, and Pengfei Luo, from the Department of Burns, Changhai Hospital, Second Military Medical University, Shanghai, China.

\section{REFERENCES}

1. Jeong W, Rhee K. Hemodynamics of cerebral aneurysms: computational analyses of aneurysm progress and treatment. Comput Mathematic Methods Med 2012;2012:782801

2. Sforza DM, Putman CM, Cebral JR. Hemodynamics of cerebral aneurysms. Annu Rev Fluid Mech 2009;41:91-107

3. Scholtes F, Martin D. Strategical implications of aneurysmal cranial nerve compression. Neuro-Chirurgie 2012;58:146-55

4. Langner S, Schroeder HW, Hosten N, et al. [Diagnosing neurovascular compression syndromes]. RoFo 2012;184:220-28

5. Shojima M, Oshima M, Takagi K, et al. Magnitude and role of wall shear stress on cerebral aneurysm: computational fluid dynamic study of 20 middle cerebral artery aneurysms. Stroke 2004;35:2500-05

6. Tremmel M, Dhar S, Levy EI, et al. Influence of intracranial aneurysm-to-parent vessel size ratio on hemodynamics and implication for rupture: results from a virtual experimental study. Neurosurgery 2009;64:622-30

7. Jou LD, Lee DH, Morsi $\mathrm{H}$, et al. Wall shear stress on ruptured and unruptured intracranial aneurysms at the internal carotid artery. AJNR Am J Neuroradiol 2008;29:1761-67
8. He $\mathrm{X}, \mathrm{Ku} \mathrm{DN}$. Pulsatile flow in the human left coronary artery bifurcation: average conditions. J Biomech Eng 1996;118:74-82

9. Dhar S, Tremmel M, Mocco J, et al. Morphology parameters for intracranial aneurysm rupture risk assessment. Neurosurgery 2008;63:185-96

10. Cebral JR, Mut F, Weir J, et al. Quantitative characterization of the hemodynamic environment in ruptured and unruptured brain aneurysms. AJNR Am J Neuroradiol 2011;32:145-51

11. Beck J, Rohde S, Berkefeld J, et al. Size and location of ruptured and unruptured intracranial aneurysms measured by 3-dimensional rotational angiography. Surg Neurol 2006;65:18-25

12. Saitua F, Acuna R, Herrera P. Percutaneous endoscopic gastrostomy: the technique of choice? J Pediatr Surg 2003;38:1512-15

13. Xiang J, Natarajan SK, Tremmel M, et al. Hemodynamic-morphologic discriminants for intracranial aneurysm rupture. Stroke 2011;42:144-52

14. Sforza DM, Putman CM, Scrivano E, et al. Blood-flow characteristics in a terminal basilar tip aneurysm prior to its fatal rupture. AJNR Am J Neuroradiol 2010;31:1127-31

15. Cebral JR, Hendrickson S, Putman CM. Hemodynamics in a lethal basilar artery aneurysm just before its rupture. AJNR Am J Neuroradiol 2009;30:95-98

16. Goubergrits L, Schaller J, Kertzscher U, et al. Statistical wall shear stress maps of ruptured and unruptured middle cerebral artery aneurysms. J R Soc Interface 2012;9:677-88

17. Takao H, Murayama Y, Otsuka S, et al. Hemodynamic differences between unruptured and ruptured intracranial aneurysms during observation. Stroke 2012;43:1436-39

18. Newby AC. Metalloproteinase expression in monocytes and macrophages and its relationship to atherosclerotic plaque instability. Arterioscler Thromb Vasc Biol 2008;28:2108-14

19. Galis ZS, Sukhova GK, Lark MW, et al. Increased expression of matrix metalloproteinases and matrix degrading activity in vulnerable regions of human atherosclerotic plaques. J Clin Invest 1994;94:2493-503

20. Gui T, Shimokado A, Sun Y, et al. Diverse roles of macrophages in atherosclerosis: from inflammatory biology to biomarker discovery. Mediat Inflamm 2012;2012:693083

21. Chiu JJ, Chien S. Effects of disturbed flow on vascular endothelium: pathophysiological basis and clinical perspectives. Physiol Rev 2011;91:327-87

22. Zeng Z, Durka MJ, Kallmes DF, et al. Can aspect ratio be used to categorize intra-aneurysmal hemodynamics? A study of elastase induced aneurysms in rabbit. J Biomech 2011;44:2809-16

23. Xu J, Yu Y, Wu X, et al. Morphological and hemodynamic analysis of mirror posterior communicating artery aneurysms. PloS One. 2013;8:e55413

24. Castro M, Putman C, Radaelli A, et al. Hemodynamics and rupture of terminal cerebral aneurysms. Acad Radiol 2009;16:1201-07 